\title{
Liderazgo transformacional y productividad en los docentes de la escuela fiscal Antonio Jose de Sucre de la provincia de Guayas - Ecuador
}

\section{Transformational leadership and productivity in the teachers of the Antonio Jose from Sucre fiscal school of the province of Guayas - Ecuador \\ ${ }^{1}{ }^{2}$}

\section{RESUMEN}

A continuación, se presenta la síntesis de la investigación "El liderazgo transformacional y productividad en los docentes de la escuela fiscal Antonio José de Sucre de la provincia de Guayas, Ecuador. El objetivo de la investigación estuvo dirigido a determinar la relación entre El liderazgo transformacional y productividad. La investigación es de tipo pura o fundamental, el alcance fue descriptivo correlacional y el diseño utilizado es no experimental, de corte transversal. La muestra fue de 50 docentes de la escuela fiscal Antonio José de Sucre de la provincia de Guayas, Ecuador Para recolectar los datos se utilizó como técnica la encuesta para la variable liderazgo transformacional y como instrumento el cuestionario que fue aplicado a los docentes, para la variable productividad se empleó como instrumento el cuestionario; en cuanto al cuestionario para dicho instrumento se realizó la confiabilidad de Alpha de Cronbach. El procesamiento de datos se realizó con el software SPSS (versión 23).

Realizado el análisis descriptivo y la correlación a través del coeficiente de Rho de Spearman, con un resultado de $\mathrm{Rho}=0.850$, interpretándose como relación entre las variables, con una $\rho=0.00(\mathrm{p}<0.05)$, con el cual se rechaza la hipótesis nula por lo tanto los resultados señalan que existe una relación directa y significativa entre el liderazgo transformacional y productividad en los docentes de la escuela fiscal Antonio José de Sucre de la provincia de Guayas, Ecuador.

Palabra clave: liderazgo transformacional, productividad, escuela, docentes

\section{ABSTRACT}

Next, the synthesis of the research "The transformational leadership and productivity in the teachers of the fiscal school Antonio José de Sucre of the province of Guayas, Ecuador. The objective of the research was aimed at determining the relationship between transformational leadership and productivity. The research is pure or fundamental, the scope was descriptive correlational, and the design used is non-experimental, cross-sectional. The sample was of 50 teachers from the fiscal school Antonio José de Sucre of the province of Guayas, Ecuador. To collect the data, the survey was used as a technique for the transformational leadership variable and as an instrument the questionnaire was applied to the teachers, for the productivity variable the questionnaire was used as an instrument; As for the questionnaire for this instrument, the reliability of Alpha de Cronbach was performed. Data processing was performed with the SPSS software (version 23).

Performed the descriptive analysis and the correlation through Spearman's Rho coefficient, with a result of Rho $=0.850$, interpreted as a relation between the variables, with a $\rho=0.00(\mathrm{p}<0.05)$, with which 
the null hypothesis is rejected therefore, the results indicate that there is a direct and significant relationship between transformational leadership and productivity in the teachers of the fiscal school Antonio José de Sucre of the province of Guayas, Ecuador.

Keywords: transformational leadership, productivity, school, teachers

\section{INTRODUCCION}

El liderazgo, ha sido un tema adoptado por diferentes disciplinas, especialmente por las ciencias administrativas, debido a la necesidad que han tenido los empleados de desarrollarlo, con el fin de gestionar las organizaciones.

Los líderes transformacionales tienen una clara visión de dónde la empresa habrá de estar en el futuro y qué es lo que habrá de hacer en el futuro. En efecto, los líderes transformacionales crean una visión innovativa, creencias fuertemente relacionadas a la misión, comunicación y articulación con los empleados, de tal forma que logren una congruencia en valores y creencias, así como una visión compartida de la misión a cumplir.

Las organizaciones educativas se encuentran inmersas en entornos competitivos donde las fronteras han desaparecido, se ha observado que la información es acumulada fácilmente y puede ser transmitida al mismo tiempo que es producida, entonces; la gestión administrativa es la conducción racional de las actividades de una organización, con o sin ánimo de lucro e implica la planeación, la dirección y el control de todas las actividades. Por lo tanto, la administración es imprescindible para la existencia, supervivencia y éxito de las organizaciones y en ella se está observando limitaciones que no posibilitan una adecuada productividad de los docentes.

Por otro lado, en Venezuela, Machado y Allison, (1996) encontraron que en el tema de la productividad en el docente en América Latina la producción del conocimiento científico, no alcanza al $1 \%$ del total Mundial, esto resalta que la relación en cuanto que la calidad de la formación académica del docente se relaciona con la productividad en el docente, y esto se ve reflejado en la poca producción de conocimiento en América latina.

Por lo antes expresado la presente investigación pretende determinar la relación entre la calidad de servicio educativo y la satisfacción de los estudiantes.

\section{Planteamiento del problema}

\section{Problema general}

- ¿Cuál es la relación entre el liderazgo transformacional y la productividad en los docentes de la escuela fiscal Antonio José de Sucres de la provincia de Guayas, Ecuador - 2017?

\section{Problemas Específicos:}

- ¿Cuál es la relación entre el compromiso prospectivo y la productividad en los docentes de la escuela fiscal Antonio José de Sucres de la provincia de Guayas, Ecuador - 2017?

- ¿Cuál es la relación entre el reconocimiento laboral y la productividad en los docentes de la escuela fiscal Antonio José de Sucres de la provincia de Guayas, Ecuador - 2017?

- ¿Cuál es la relación entre la responsabilidad funcional y la productividad en los docentes de la escuela fiscal Antonio José de Sucres de la provincia de Guayas, Ecuador - 2017?

- ¿Cuál es la relación entre la motivación institucional y la productividad en los docentes de la escuela fiscal Antonio José de Sucres de la provincia de Guayas, Ecuador - 2017? 


\section{Objetivo general}

- Determinar la relación entre el liderazgo transformacional y la productividad en los docentes de la escuela fiscal Antonio José de Sucres de la provincia de Guayas, Ecuador - 2017

\section{Objetivos específicos}

- Evidenciar la relación entre el compromiso prospectivo y la productividad en los docentes de la escuela fiscal Antonio José de Sucres de la provincia de Guayas, Ecuador - 2017

- Evidenciar la relación entre el reconocimiento laboral y la productividad en los docentes de la escuela fiscal Antonio José de Sucres de la provincia de Guayas, Ecuador - 2017

- Evidenciar la relación entre la responsabilidad funcional y la productividad en los docentes de la escuela fiscal Antonio José de Sucres de la provincia de Guayas, Ecuador - 2017

- Evidenciar la relación entre la motivación institucional y la productividad en los docentes de la escuela fiscal Antonio José de Sucres de la provincia de Guayas, Ecuador - 2017.

\section{Hipótesis general}

Hipótesis general alterna (HA)

- Existe relación significativa entre el liderazgo transformacional y la productividad en los docentes de la escuela fiscal Antonio José de Sucres de la provincia de Guayas, Ecuador - 2017

Hipótesis general nula $(\mathrm{HO})$

- No existe relación significativa entre el liderazgo transformacional y la productividad en los docentes de la escuela fiscal Antonio José de Sucres de la provincia de Guayas, Ecuador - 2017

\section{Hipótesis especifica}

Hipótesis alterna (HA)1

- Existe relación significativa entre el compromiso prospectivo y la productividad en los docentes de la escuela fiscal Antonio José de Sucres de la provincia de Guayas, Ecuador - 2017

Hipótesis nula (HO) 1

- No existe relación significativa entre el compromiso prospectivo y la productividad en los docentes de la escuela fiscal Antonio José de Sucres de la provincia de Guayas, Ecuador - 2017

Hipótesis alterna (HA)2

- Existe relación significativa entre el reconocimiento laboral y la productividad en los docentes de la escuela fiscal Antonio José de Sucres de la provincia de Guayas, Ecuador - 2017

Hipótesis general nula ( $\mathrm{HO})$

- No existe relación significativa entre el reconocimiento laboral y la productividad en los docentes de la escuela fiscal Antonio José de Sucres de la provincia de Guayas, Ecuador - 2017

Hipótesis alterna (HA)3

- Existe relación significativa entre la responsabilidad funcional y la productividad en los docentes de la escuela fiscal Antonio José de Sucres de la provincia de Guayas, Ecuador - 2017

Hipótesis general nula (HO)

- No existe relación significativa entre la responsabilidad funcional y la productividad en los docentes de la escuela fiscal Antonio José de Sucres de la provincia de Guayas, Ecuador - 2017

Hipótesis alterna (HA)4

- Existe relación significativa entre la motivación institucional y la productividad en los docentes de la escuela fiscal Antonio José de Sucres de la provincia de Guayas, Ecuador - 2017 
Hipótesis general nula $(\mathrm{HO})$

- No existe relación significativa entre la motivación institucional y la productividad en los docentes de la escuela fiscal Antonio José de Sucres de la provincia de Guayas, Ecuador - 2017

\section{MATERIAL Y MÉTODOS}

Objeto del estudio:

Estuvo conformado por profesores de escuela fiscal Antonio José de Sucre

Población:

La población se constituyó de 50 docentes escuela fiscal Antonio José de Sucre

Muestra:

La muestra se constituyó de 50 docentes de la escuela fiscal Antonio José de Sucre

Instrumentos de recolección de datos:

En el estudio se hizo uso de la técnica de la Encuesta, por la modalidad de estudio y el tiempo de aplicación, al respecto se utilizó el instrumento cuestionario.

Métodos y Técnicas:

Método hipotético deductivo en tal sentido, de acuerdo con Hernández et al (2010) la encuesta es el procedimiento adecuado para recolectar datos a grandes muestras en un solo momento, de ahí que en este estudio se asume dicha técnica ya que como muestra se trabajará con un total de 50 docentes.

Técnica

Encuesta

Diseño de estudio:

El diseño corresponde a la Investigación correlacional. Sánchez y Reyes (2002) al respecto manifiestan que este diseño "se orienta a la determinación del grado de relación existente entre dos o más variables de interés en una misma muestra de sujeto o el grado de relación entre dos fenómenos o eventos observados". (p. 79)

Procedimiento:

- Se coordina con la organización para la toma de las encuestas

- Se elaboró las encuestas referidas a cada variable

- Después se aplicó las encuestas

- Posteriormente la data se procesaron, analizaron y se discutieron

- Se redactó las conclusiones y sugerencias en base a las discusiones de los resultados

- Se comunicaron las conclusiones del trabajo de investigación.

\section{Analisis, interpretacion de resultados}

Tabla $N^{\circ} 1$. Niveles de la variable liderazgo transformacional

\begin{tabular}{cccc} 
& & Frecuencia & Porcentaje \\
\multirow{3}{*}{ Válido } & Bajo & 3 & 6,0 \\
& Regular & 20 & 40,0 \\
& Alto & 27 & 54,0 \\
& Total & 50 & 100,0
\end{tabular}

Fuente: Base de datos 


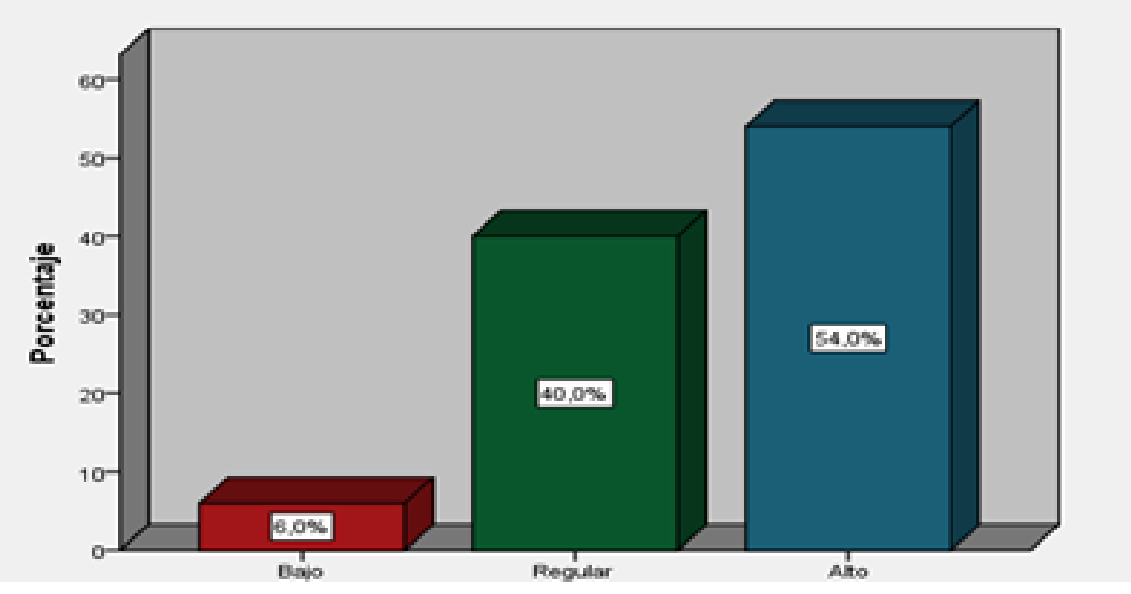

Figura $\mathrm{N}^{\circ} 1$. Niveles de la variable liderazgo transformacional

En la tabla y figura, muestra que de los 50 docentes encuestados, el 6\% consideran que esta en un nivel bajo el liderazgo transformacional que ejerce el equipo directivo e $\mathrm{n}$ la escuela fiscal Antonio José de Sucres de la provincia de Guayas, Ecuador, mientras el $40 \%$ lo considera en un nivel regular y el $54 \%$ en un nivel alto.

\section{Tabla $N^{\circ} 2$. Niveles de la dimensión compromiso prospectivo}

\begin{tabular}{cccc} 
& & Frecuencia & Porcentaje \\
\multirow{3}{*}{ Válido } & Bajo & 5 & 10,0 \\
& Regular & 20 & 40,0 \\
& Alto & 25 & 50,0 \\
& Total & 50 & 100,0
\end{tabular}

Fuente: Base de datos de excell

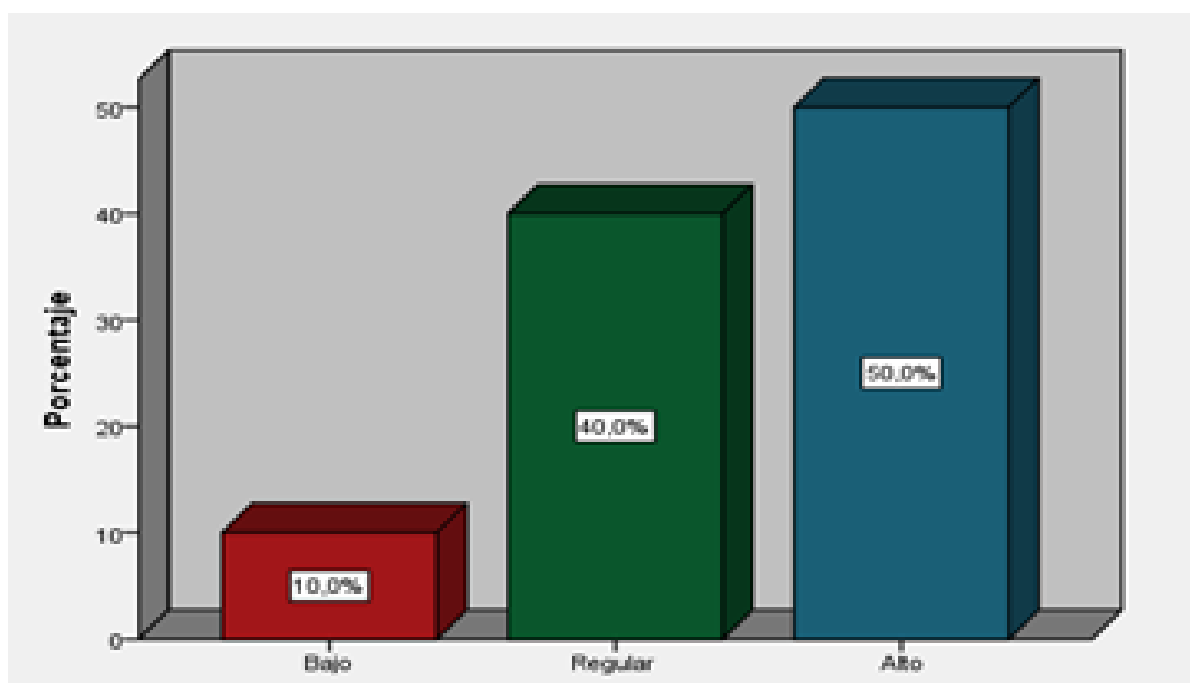

Figura $\mathrm{N}^{\circ}$ 2. Niveles de la dimensión compromiso prospectivo 
En la tabla y figura, muestra que de los 50 docentes encuestados, el 10\% consideran que esta en un nivel bajo en cuanto a la dimensión compromiso prospectivo del liderazgo transformacional que ejerce el equipo directivo en la escuela fiscal Antonio José de Sucres de la provincia de Guayas, Ecuador, mientras el $40 \%$ lo considera en un nivel regular y el 50\% en un nivel alto.

Tabla $\mathrm{N}^{\circ}$ 3. Niveles de la dimensión reconocimiento laboral

\begin{tabular}{cccc} 
& & Frecuencia & Porcentaje \\
\multirow{3}{*}{ Válido } & Bajo & 4 & 8,0 \\
& Regular & 26 & 52,0 \\
& Alto & 20 & 40,0 \\
& Total & 50 & 100,0
\end{tabular}

Fuente: Base de datos de excell

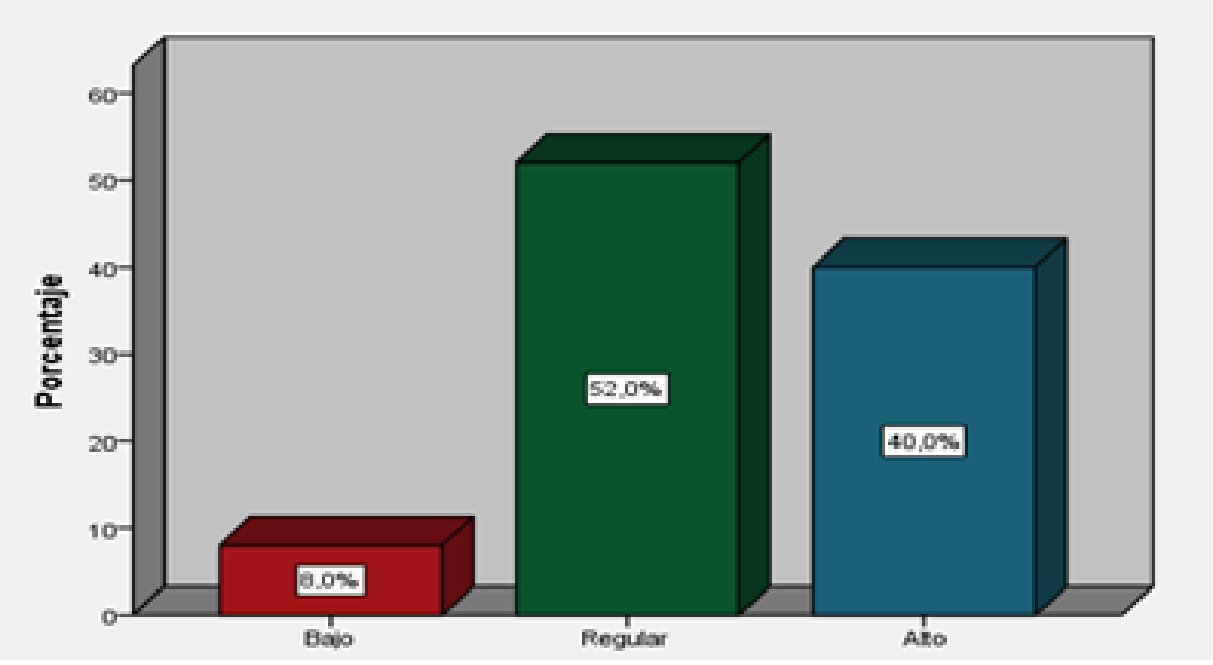

Figura $\mathrm{N}^{\circ}$ 3. Niveles de la dimensión reconocimiento laboral

En la tabla y figura, muestra que de los 50 docentes encuestados, el $8 \%$ consideran que esta en un nivel bajo en cuanto a la dimensión reconocimiento laboral del liderazgo transformacional que ejerce el equipo directivo en la escuela fiscal Antonio José de Sucres de la provincia de Guayas, Ecuador, mientras el 52\% lo considera en un nivel regular y el $40 \%$ en un nivel alto.

Tabla $\mathrm{N}^{\circ}$ 4. Niveles de la dimensión responsabilidad funcional

\begin{tabular}{llcc} 
& & Frecuencia & Porcentaje \\
\multirow{3}{*}{ Válido } & Bajo & 7 & 14,0 \\
& Regular & 20 & 40,0 \\
& Alto & 23 & 46,0 \\
& Total & 50 & 100,0
\end{tabular}

Fuente: Base de datos de excell 


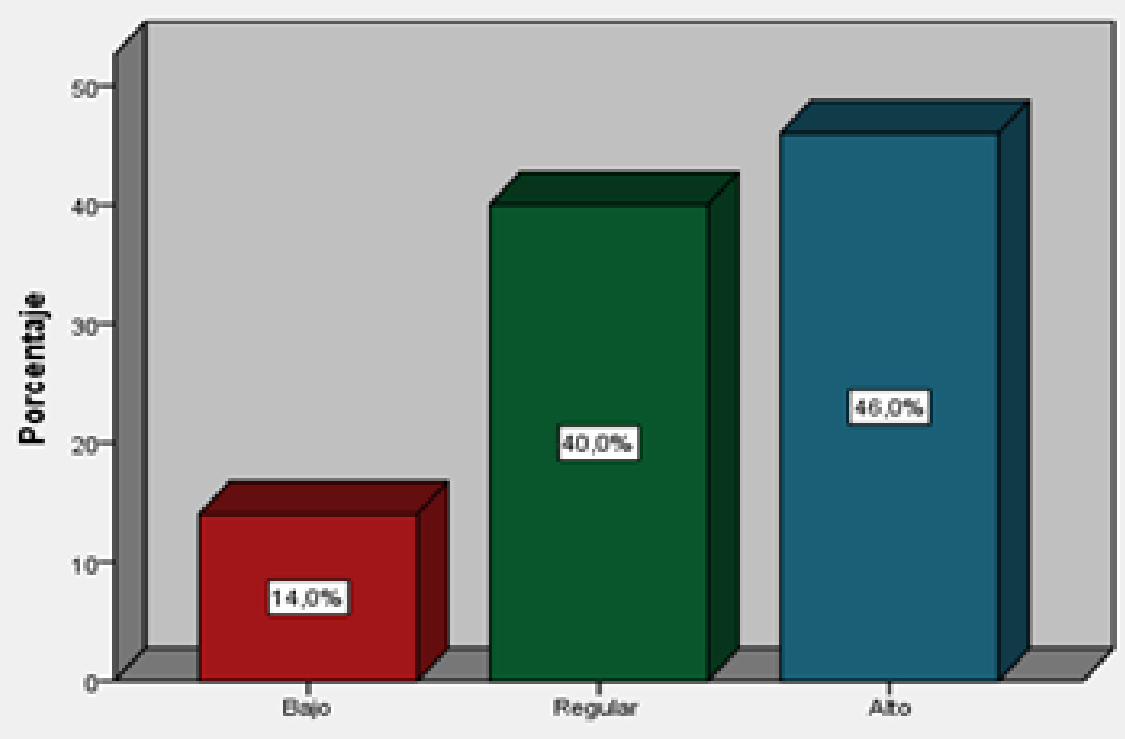

Figura $\mathrm{N}^{\circ}$ 4. Niveles de la dimensión responsabilidad funcional

En la tabla y figura, muestra que de los 50 docentes encuestados, el 14\% consideran que esta en un nivel bajo en cuanto a la dimensión responsabilidad funcional del liderazgo transformacional que ejerce el equipo directivo en la escuela fiscal Antonio José de Sucres de la provincia de Guayas, Ecuador, mientras el $40 \%$ lo considera en un nivel regular y el $46 \%$ en un nivel alto.

\section{Tabla $\mathrm{N}^{\circ}$ 5. Niveles de la dimensión motivación intelectual}

\begin{tabular}{llcc} 
& & Frecuencia & Porcentaje \\
\multirow{3}{*}{ Válido } & Bajo & 4 & 8,0 \\
& Regular & 26 & 52,0 \\
& Alto & 20 & 40,0 \\
& Total & 50 & 100,0
\end{tabular}

Fuente: Base de datos de excell

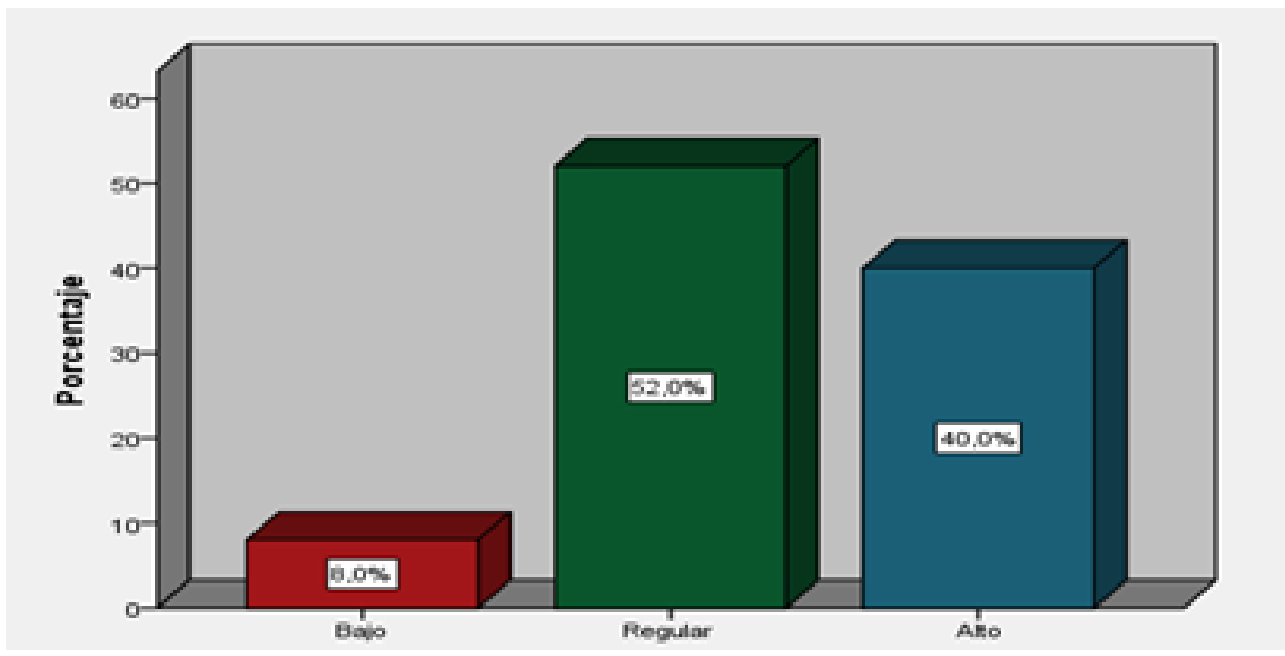

Figura $\mathrm{N}^{\circ}$ 5. Niveles de la dimensión motivación intelectual 
En la tabla y figura, muestra que de los 50 docentes encuestados, el $8 \%$ consideran que esta en un nivel bajo en cuanto a la dimensión motivación intelectual del liderazgo transformacional que ejerce el equipo directivo en la escuela fiscal Antonio José de Sucres de la provincia de Guayas, Ecuador, mientras el 52\% lo considera en un nivel regular y el $40 \%$ en un nivel alto.

Tabla $N^{\circ}$ 6. Niveles de la variable productividad en los docentes

\begin{tabular}{llcc} 
& & Frecuencia & Porcentaje \\
\multirow{4}{*}{ Válido } & Inadecuada & 2 & 4,0 \\
& Regular & 18 & 36,0 \\
& Adecuada & 30 & 60,0 \\
& Total & 50 & 100,0
\end{tabular}

Fuente: Base de datos de excell

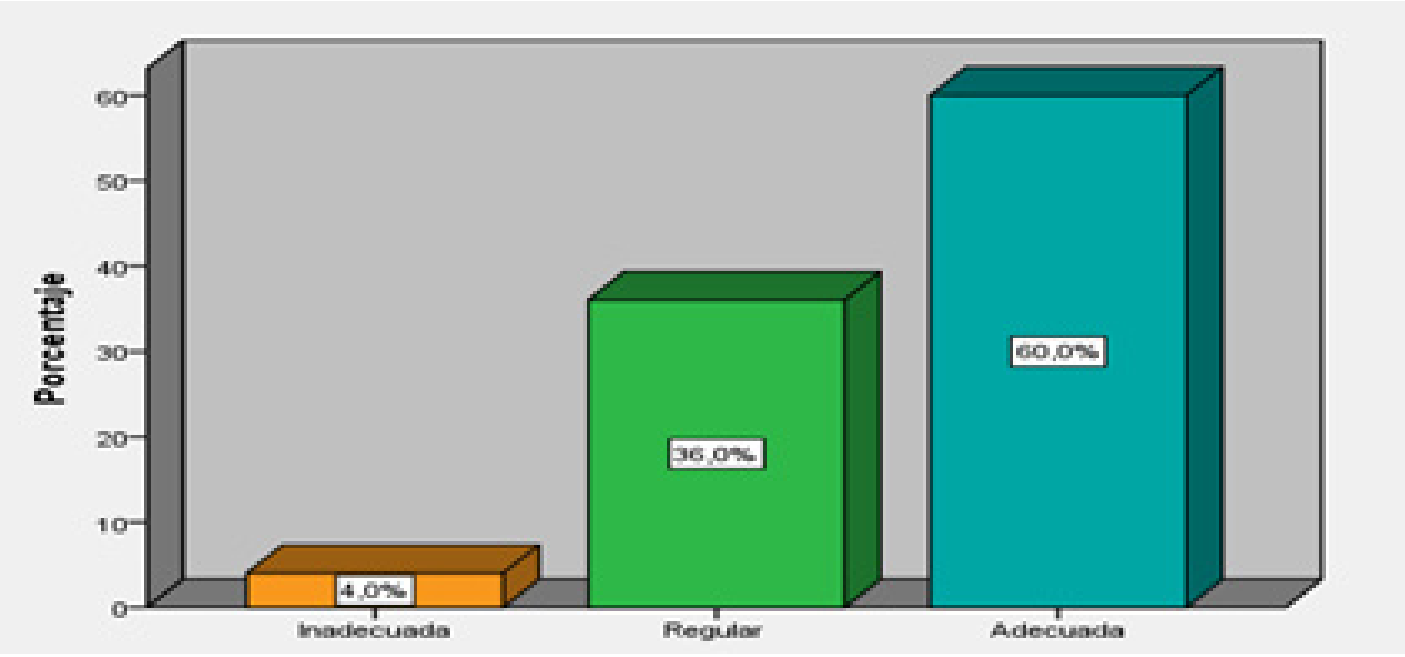

Figura $\mathrm{N}^{\circ}$ 6. Niveles de la variable productividad en los docentes

En la tabla y figura, muestra que de los 50 docentes encuestados, el $4 \%$ consideran que esta en un nivel bajo en cuanto a la productividad en los docentes en la escuela fiscal Antonio José de Sucres de la provincia de Guayas, Ecuador, mientras el 36\% lo considera en un nivel regular y el $60 \%$ en un nivel alto.

Tabla $N^{\circ} 7$. Niveles de la dimensión factores higiénicos

\begin{tabular}{llcc} 
& & Frecuencia & Porcentaje \\
\multirow{3}{*}{ Válido } & Inadecuada & 3 & 6,0 \\
& Regular & 18 & 36,0 \\
& Adecuada & 29 & 58,0 \\
& Total & 50 & 100,0
\end{tabular}

Fuente: Base de datos de excell 


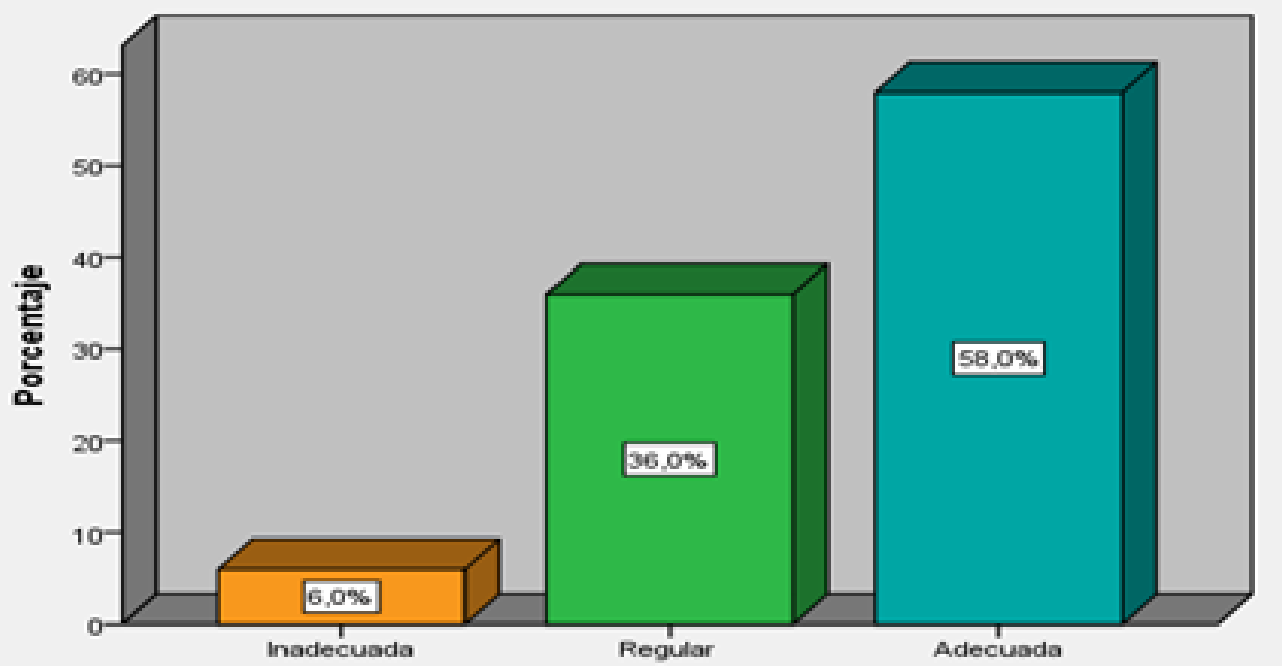

Figura $\mathrm{N}^{\circ}$ 5. Niveles de la dimensión factores higiénicos

En la tabla y figura, muestra que de los 50 docentes encuestados, el 6\% consideran que esta en un nivel bajo en cuanto a la dimensión factores higiénicos en la escuela fiscal Antonio José de Sucres de la provincia de Guayas, Ecuador, mientras el 36\% lo considera en un nivel regular y el 58\% en un nivel alto.

\section{Tabla $\mathrm{N}^{\circ}$ 8. Niveles de la dimensión factores motivacionales}

\begin{tabular}{|llcc}
\multirow{4}{*}{ Válido } & & Frecuencia & Porcentaje \\
& Inadecuada & 1 & 2,0 \\
& Regular & 20 & 40,0 \\
& Adecuada & 29 & 58,0 \\
& Total & 50 & 100,0
\end{tabular}

Fuente: Base de datos de excell

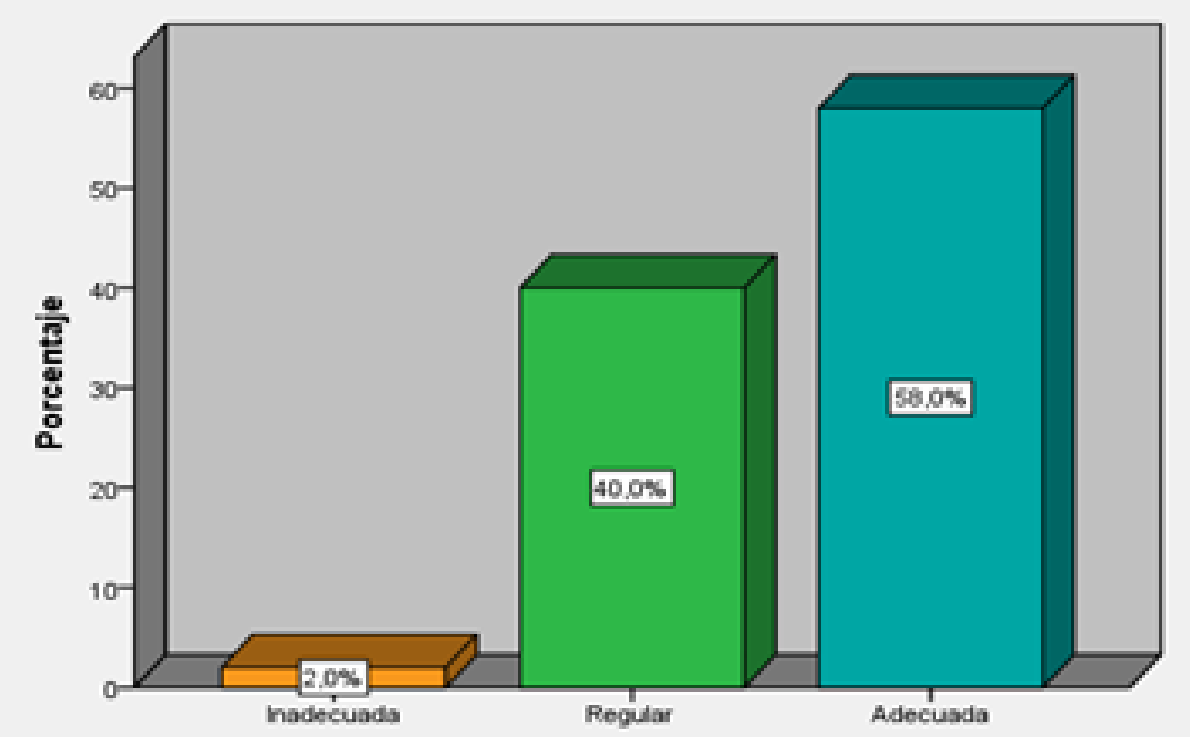

Figura $\mathrm{N}^{\circ}$. Niveles de la dimensiones factores motivacionales 
En la tabla y figura, muestra que de los 50 docentes encuestados, el $2 \%$ consideran que esta en un nivel bajo en cuanto a la dimensión factores motivacionales en la escuela fiscal Antonio José de Sucres de la provincia de Guayas, Ecuador, mientras el 40\% lo considera en un nivel regular y el 58\% en un nivel alto.

\section{DISCUSIÓN}

En la presente tesis se pudo analizar y afirmar que existe relación significativa entre el liderazgo transformacional y la productividad en los docentes de la escuela fiscal Antonio José de Sucre de la provincia de Guayas, Ecuador - 2017, con un coeficiente de correlación de Rho de Spearman $=0,850^{* *}$; al respecto la investigación realizada por Carbone, (2008) sobre Situación de liderazgo educativo en Chile concluyeron en que el Liderazgo Transformacional, en la que resalta que es un factor fundamental e importante para el éxito de la gestión educativa en las escuelas llegando a proponer un perfil del director y a concluir que los resultados son buenos cuando se trata de directivos participativos y son malos resultados cuando hay presencia de un equipo directivo centralista.

Así mismo la investigación que realizo Castillo, (2010), sobre "Relación del liderazgo de la directora y el desempeño laboral" en la cual se llegó a establecer la no existencia de relación entre el estilo de liderazgo de la directora con el desempeño de sus docentes, eso ha hecho que se genere problemas a nivel institucional.

En cuanto a las hipótesis especifica primera se pudo afirmar que existe relación significativa entre el compromiso prospectivo y la productividad en los docentes de la escuela fiscal Antonio José de Sucre de la provincia de Guayas, Ecuador - 2017, con un coeficiente de correlación de Rho de Spearman $=0,801^{* *}$; al respecto la investigación realizada por Ávila y Velásquez (2014) sobre liderazgo transformacional como herramienta de la productividad, en la cual se concluyó que el liderazgo es el proceso de influencia de líderes y seguidores para alcanzar los objetivos de la organización. se hizo una propuesta dando sugerencias a cada uno de los elementos que se consideraron más relevantes en un líder trasformador.

En la segunda hipótesis se pudo afirmar que existe relación significativa entre el reconocimiento laboral y la productividad en los docentes de la escuela fiscal Antonio José de Sucre de la provincia de Guayas, Ecuador - 2017, con un coeficiente de correlación de Rho de Spearman $=0,730^{\star *}$; en su investigación Balda (2015) sobre liderazgo educativo transformacional como necesidades de las instituciones educativas en la republica de Ecuador; en la que se concluye que el cambio de los estilos de dirección al superar el tradicional liderazgo educativo del director favorece que las instituciones educativas sean más pertinentes con sus comunidades y entornos a partir de un accionar conjunto que integre a las familias, instituciones públicas y privadas y organizaciones en el enfrentamiento a cada manifestación no coherente con la formación ciudadana que forma parte de la misión de estas instituciones educativas.

La tercera hipótesis planteada se pudo afirmar que existe relación significativa entre la responsabilidad funcional y la productividad en los docentes de la escuela fiscal Antonio José de Sucre de la provincia de Guayas, Ecuador - 2017, con un coeficiente de correlación de Rho de Spearman $=0,744^{* *}$, al respecto Ramírez (2016) realizó la tesis titulada Calidad de formación académica y productividad en el docente; en la que concluyo que existe una correlación media entre la calidad de formación académica y productividad en el docentes.

Finalmente se pudo afirmar que existe relación significativa entre la motivación institucional y la productividad en los docentes de la escuela fiscal Antonio José de Sucre de la provincia de Guayas, Ecuador - 2017, con un coeficiente de correla- 
ción de Rho de Spearman $=0,678^{* *}$, al respecto Gamba (2015) en su investigación titulada La motivación y su relación con la productividad laboral, señalo que para la motivación encontramos que el ambiente de trabajo es ideal para desarrollar las tareas. Para la motivación encontramos que el ambiente de trabajo es ideal para desarrollar las tareas.

\section{CONCLUSIONES}

\section{PRIMERA:}

De acuerdo a las evidencias estadísticas, se puede afirmar que existe relación significativa entre el liderazgo transformacional y la productividad en los docentes de la escuela fiscal Antonio José de Sucre de la provincia de Guayas, Ecuador - 2017, ya que el nivel de significancia calculada es $\mathrm{p}<$ .05 y el coeficiente de correlación Rho de Spear$\operatorname{man}=0,850^{\star *}$.

\section{SEGUNDA:}

En cuanto a la hipótesis especifica segunda se evidencio que existe relación significativa entre el compromiso prospectivo y la productividad en los docentes de la escuela fiscal Antonio José de Sucre de la provincia de Guayas, Ecuador - 2017, ya que el nivel de significancia calculada es $\mathrm{p}<$ .05 y el coeficiente de correlación Rho de Spear$\operatorname{man}=0,801^{\star *}$.

\section{TERCERA:}

En cuanto a la hipótesis especifica tercera se evidencio que existe relación significativa entre el reconocimiento laboral y la productividad en los docentes de la escuela fiscal Antonio José de Sucre de la provincia de Guayas, Ecuador - 2017, ya que el nivel de significancia calculada es $\mathrm{p}<.05$ y el coeficiente de correlación Rho de Spearman $=0,744^{*}$

\section{CUARTA:}

En referencia a la tercera hipótesis especifica se mostró que existe relación significativa entre la responsabilidad funcional y la productividad en los docentes de la escuela fiscal Antonio José de Sucre de la provincia de Guayas, Ecuador - 2017, se obtuvo un coeficiente de correlación de Rho de Spearman $=0,744^{* *}$, con una $\rho=0.000(\rho<$ 0.05).

\section{QUINTA:}

En referencia a la cuarta hipótesis especifica se mostró que existe relación significativa entre la motivación institucional y la productividad en los docentes de la escuela fiscal Antonio José de Sucre de la provincia de Guayas, Ecuador - 2017, se obtuvo un coeficiente de correlación de Rho de Spearman $=0,678^{\star *}$, con una $\rho=0.000(\rho<$ 0.05).

\section{REFERENCIAS}

Ávila Y y Velásquez M (2014) Liderazgo transformacional como herramienta de la productividad (tesis de grado) Universidad Autónoma del Estado de México.

Adam, E., Hershauer, J. y Ruch, W. (2001). Productividad y Calidad. Su medición como base del mejoramiento. México D.F.: Trillas

Balda R (2015) Liderazgo educativo transformacional como necesidades de las instituciones educativas en la republica de Ecuador. Revista electrónica formación y calidad educativa REFCalE. 3 (2)

Burns, J. (1978). "Leadership", Nueva York, Harper\& Row

Bass, B. (1985) "Liderazgo y comportamiento en función a las Expectativas”. México: Interamericana

Bass, B. (2000) "Liderazgo y organizaciones que aprenden”. ICE Deusto. Actas del III Congreso internacional sobre dirección de centros educativos.

Bryman, A. (1996). "Charisma \& leadership in organization”. London, Sage publications Bolívar, A. (1997). "Liderazgo, mejora y centros educativos". El Liderazgo en Educación,

Cannice. M., Koontz. H. y Weihrich. H. (2012). Administración una Perspectiva Global empresarial. México. 14 a edición. Pp.412-419

Carbone, R. (2008). Situación de Liderazgo Educativo en Chile. Santiago, Chile. Universidad Alberto Hurtado.

Cequea, M., Rodríguez, C. y Núñez, M. (2011). La productividad desde una perspectiva humana: dimensiones y factores. Intangible Capital, 7(2): 549-584. 
Cosío, M. (2005) Teorías del liderazgo Mc Graw Hill. Bogotá Colombia.

Crespo, G. (2013). Productividad. Consultado el 20 de agosto del 2016. Recuperado en: http:/www.gedesco.es/ blog/definicion-de-productividad/

Christensen, G. \& Walters, H. (2007) “El líder en la organización” Paidos Ibérica. Madrid

Chiavenato, I. (1993). Introducción a la teoría general de la Administración. México: McGraw-Hill.

Chiavenato, I. (2006) “Talento humano”. México. Interamericana. Prentice Hall.

Gallo A (2015) Análisis del liderazgo institucional en la gestión escolar de la institución educativa "Santa María Mazarello" Universidad Politécnica Salesiana sede Quito. Ecuador

García, I. (2006).” La formación del clima sicológico y su relación con los estilos de liderazgo". Granada, España: Universidad de Granada. Tesis doctoral.

Dellepiane, L. (2004). Liderazgo México: Fondo de cultura económica

Ferreiro, P y Alcázar, M. (2005). Gobierno de personas en la empresa. Lima - Perú. PAD. Escuela de Dirección Universidad de Piura

Fuentes, S. (2012). Satisfacción laboral y su influencia en la productividad. (Tesis de maestría). Quetzaltenango: Universidad Rafael Landívar.

Gamba, J. (2015). La motivación y su relación con la productividad. (Tesis de maestría). México: Universidad Autónoma de Querétaro.

Hernández, R. Fernández C. y Batista, L. (2014). Metodología de la Investigación. ( $5^{\text {a }}$ ed).México: Mc Graw-Hill Interamericana.

Hoyle, R. (2004) Administración. México: Mc Graw Hill Jason, P. (2005) Comportamiento organizacional México. Trillas

Jones, G. \& George, J. (2006) “Administración contemporánea" 4ta. Edición México. D.F. Mc Graw Hill Interamericana

Leithwood, K. (1999). "Efectos del Liderazgo Transformador, una perspectiva global". Colombia. Mc Graw Hill
López, P (2014) El liderazgo transformacional como agente motivador en un establecimiento municipal Universidad de Chile tesis de doctorado

Martins, F., Carmmaroto, A., Neris, L., \& Canelón, E. (2009). Liderazgo transformacional y gestión educativa en contextos descentralizados. Actualidades investigativas en educación http://revista.inie.ucr.ac.cr. Volumen 9, Número 2, Año 2009, ISSN 1409-4703

Morocho, L. (2010) “El liderazgo Transformacional y el clima Organizacional de las I.E. de la ciudad Satélite Santa Rosa Región Callao" Universidad San Ignacio de Loyola Lima. Tesis de maestría.

Quiroa, C. (2014). Toma de decisiones y productividad laboral. (Tesis de pregrado). Quetzaltenango: Universidad Rafael Landívar

Ramírez, M. (2016). Calidad de formación académica y productividad de los docentes de la escuela académica profesional de marketing y negocios internacionales de la universidad de Huánuco - 2016. (Tesis de Maestría, Universidad de Huánuco).

Robbins, S., y Coulter, M. (2010). Administración. Décima edición. México: Prentice-Hall

Rojas, A. \& Gaspar, G. (2006). "Bases del liderazgo en educación” Santiago. Chile: OREALC / UNESCO

Salazar, M. (2006). El liderazgo transformacional ¿modelo para organizaciones educativas que aprenden? UNIrevista. Vol 1, $\mathbf{N}^{\circ}$ 3. Universidad Viña del Mar. Chile.

Singh, H. (2008). Human factor and organisational productivity in textile industry and agricultural machinery industry in north India. (Doctoral thesis). India: Panjab University, Chandigarh

Stogdill, R. (2001). “Comportamiento Organizacional”. Mexico: Trillas

Vázquez, A. (2013). Interdependencia entre el liderazgo transformacional, cultura organizacional y cambio educativo: una reflexión. Revista Iberoamericana sobre Calidad, Eficacia y cambio en Educación.11 (73)

Vilar, L. (2006). "Liderazgo y organizaciones que aprenden”. Bilbao: Mensajero

Warren, J. (2006) Liderazgo México. Fondo de cultur 